\title{
Mechanisms of critical period in the hippocampus underlie object location learning and memory in infant rats
}

\author{
Alessio Travaglia, ${ }^{1}$ Adam B. Steinmetz, ${ }^{1}$ Janelle M. Miranda, ${ }^{1}$ and Cristina M. Alberini ${ }^{1,2}$ \\ ${ }^{1}$ Center for Neural Science, New York University, New York, New York 10003, USA; ${ }^{2}$ Neuroscience Institute, NYU Langone Medical \\ Center, New York, New York 10016, USA
}

\begin{abstract}
Episodic memories in early childhood are rapidly forgotten, a phenomenon that is associated with "infantile amnesia," the inability of adults to remember early-life experiences. We recently showed that early aversive contextual memory in infant rats, which is in fact rapidly forgotten, is actually not lost, as reminders presented later in life reinstate a long-lasting and context-specific memory. We also showed that the formation of this infantile memory recruits in the hippocampus mechanisms typical of developmental critical periods. Here, we tested whether similar mechanisms apply to a nonaversive, hippocampal type of learning. We report that novel object location (nOL) learned at postnatal day 17 (PNI7) undergoes the typical rapid forgetting of infantile learning. However, a later reminder reinstates memory expression. Furthermore, as for aversive experiences, nOL learning at PN17 engages critical period mechanisms in the dorsal hippocampus: it induces a switch in the GluN2A/2B-NMDA receptor ratio, and brain-derived neurotrophic factor injected bilaterally into the dorsal hippocampus immediately after training results in long-lasting memory expression. We conclude that in infancy the hippocampus plays a necessary role in processing episodic and contextual memories, including nonaversive ones, and matures through a developmental critical period.
\end{abstract}

Episodic memories formed during the first postnatal period are rapidly forgotten, a phenomenon associated with infantile amnesia, the inability of adults to recall early-life memories (Campbell and Spear 1972; Rovee-Collier 1999; Hayne 2004). This rapid forgetting is found in humans as well as in nonhuman animals, suggesting that infantile amnesia occurs throughout evolution (Campbell and Spear 1972; Rovee-Collier 1999; Hayne 2004). Despite being rapidly forgotten, childhood experiences appear to influence brain functions throughout life (Heim and Nemeroff 2001). The reasons for this paradox have been debated: some authors suggested that infantile amnesia is the result of the immaturity of the infant brain, hence its inability to be functionally competent (Campbell and Spear 1972; Nelson 2000; Dumas and Rudy 2010; Akers et al. 2014); others indicated that the infantile memories, although apparently forgotten, are not gone, and suggested that the amnesia reflects retrieval impairment (Spear and Parsons 1976; Kim et al. 2006; Li et al. 2014). We recently identified novel mechanisms associated to early-life episodic experiences and offered a new explanation for infantile amnesia. In line with several previous studies (Campbell and Campbell 1962; Rudy and Morledge 1994; Li et al. 2014), we showed the rapid forgetting typical of infantile amnesia in PN17 rats using the aversive contextual learning paradigm inhibitory avoidance (IA). We then found that the forgotten memory is reinstated following reminders given later in life; the reinstated memory is long-lasting and context-specific, indicating that training at PN17 produces a long-term, latent memory trace (Travaglia et al. 2016a). In contrast to what was previously believed, we found that the role of the dorsal hippocampus is necessary for the formation of this latent memory. More specifically this memory requires the function of the metabotropic glutamate receptor 5 (mGluR5)-

Corresponding author: ca60@nyu.edu

Article is online at http://www.learnmem.org/cgi/doi/10.1101//m.046946. 117. and brain-derived neurotrophic factor (BDNF)-dependent switch in the ratio GluN2A/GluN2B (Travaglia et al. 2016a) in the dorsal hippocampus. These molecular mechanisms are typical of developmental critical periods, which are temporally limited phases during which brain systems are especially sensitive to stimuli, and experience organizes their functional development and permanently alters their performance (Hensch 2005). We also found that, as during the visual system critical period (Hanover et al. 1999; Huang et al. 1999), an increase in BDNF is sufficient to promote functional and behavioral maturation: in fact, a bilateral hippocampal injection of BDNF immediately after training at PN17 produces the expression of long-term memory (Travaglia et al. 2016a). These results led us to propose that the hippocampus, like sensory cortices, undergoes a critical period during which it matures in response to learning experience (Travaglia et al. 2016a). This new view reconciles the debates of how infantile memories, while not being functionally mature, can exert a critical influence on learning and memories throughout life (Alberini and Travaglia 2017).

Previous studies have shown that nonaversive episodic memories in animal models, such as novel object location (nOL), novel object recognition, and spatial memories undergo rapid forgetting and infantile amnesia (Anderson et al. 2004; Reger et al. 2009; Krüger et al. 2012; Jablonski et al. 2013; Westbrook et al. 2014). However, very little is known about their underlying mechanisms and, specifically, it remains to be determined whether these memories, like aversive ones, engage mechanisms of developmental

\footnotetext{
(C) 2018 Travaglia et al. This article is distributed exclusively by Cold Spring Harbor Laboratory Press for the first 12 months after the full-issue publication date (see http://learnmem.cshlp.org/site/misc/terms.xhtml). After 12 months, it is available under a Creative Commons License (Attribution-NonCommercial 4.0 International), as described at http://creativecommons.org/licenses/by-nc/ $4.0 \%$.
} 
critical period. To address these questions, here we used the nOL paradigm (Ennaceur et al. 1997; Gerstein et al. 2013), which, based on rodents' natural tendency to explore novel stimuli and places, measures the memory of a previously encountered object's spatial location (Ennaceur et al. 1997; Gerstein et al. 2013). This learning is nonaversive and requires the function of the hippocampus in adult rodents (Mumby et al. 2002; Gaskin et al. 2009; Warburton and Brown 2015). We compared memory formation and retention in infant rats at postnatal day 17 (PN17) and at PN24, and tested whether mechanisms typical of critical period underlie memories formed at PN17.

\section{Results}

nOL memory learned at PN17 is rapidly forgotten

To test the persistence of nOL memory during early development, we measured learning and memory retention of rats trained at either PN17 or PN24 (Fig. 1). These two ages were chosen because they correspond to different phases of hippocampal maturation. According to contextual aversive learning paradigms, these ages in fact correspond to the infantile amnesia period and a more mature age at which long-term memory is expressed and persists, respectively (Campbell and Spear 1972; Rudy and Morledge 1994; Schiffino et al. 2011; Travaglia et al. 2016a; Guskjolen et al. 2017).

We found that during training, both PN17 and PN24 rats explored the objects at chance level [one samples $t$-test comparing: PN17 versus chance $(50 \%), t_{(9)}=0.58 P=0.58$, PN24 versus chance $(50 \%), t_{(8)}=0.46 P=0.66$ ] (Fig. $\left.2 \mathrm{~A}\right)$. When tested $1 \mathrm{~min}$ after training both age groups had significant memory (Fig. 2B), as revealed by the preferential exploration of the moved object (PN17: $67.3 \% \pm 3.1 \%$, one samples $t$-test comparing PN17 versus chance $(50 \%), t_{(9)}=5.58 P=0.0003$, and PN24: $68.8 \% \pm 4.7 \%$, one samples $t$-test comparing PN24 versus chance $\left.(50 \%), t_{(8)}=4.04 P=0.0038\right]$. Statistical analyses comparing the retention of PN17- and PN24-trained rats at 1 min after training showed no statistical difference between the two groups (unpaired two-tailed Student's $t$-test comparing $\%$ preference of at $1 \mathrm{~min}$ Test, $P=0.79$ ). Furthermore, the total time spent exploring the two objects did not differ across the two age groups during either training (Fig. 2C) or testing (Fig. 2D) [unpaired two-tailed Student's $t$-test comparing PN17 versus PN24 at: training $(P=0.72)$ and at 1 min test $(P=0.45)]$.

Thus, these results, in agreement with previous data on novel object recognition of PN18 rats (Anderson et al. 2004), extend to nonaversive memories the conclusion that rats trained in hippocampus-dependent tasks at PN17, during the infantile amnesia period, can acquire and express memories when tested shortly after training.

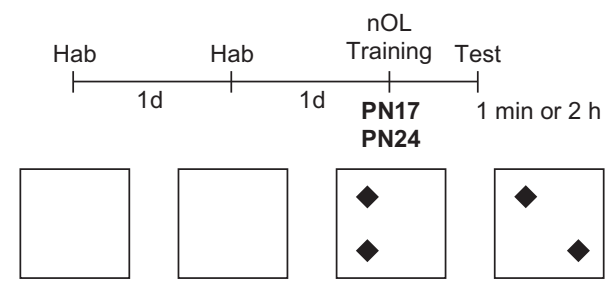

Figure 1. Schedule and schematic representation of nOL. Rats were habituated (Hab) to the arena for 5 min per day for two consecutive days (d). One day later, the rats were trained and either $1 \mathrm{~min}$ or $2 \mathrm{~h}$ after training they were tested in the same arena, where the placement of one object was changed.
Training

A

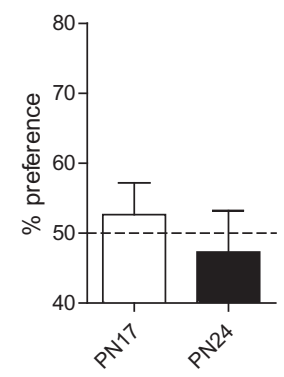

C

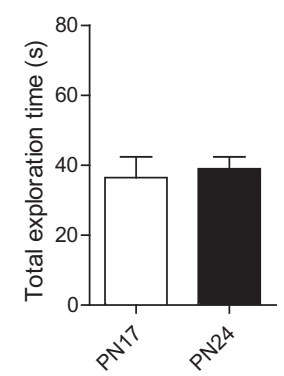

1 minute Test

B

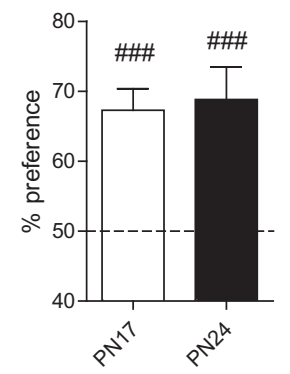

D

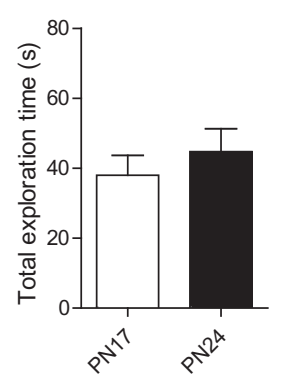

Figure 2. Rats trained at PN17 or PN24 have significant memory retention for $\mathrm{nOL} 1 \mathrm{~min}$ after training. $(A, B)$ Percent exploration time of the moved object for PN17 (white bar) and PN24 rats (black bar) at: $(A)$ training and $(B) 1 \mathrm{~min}$ after training (1 min Test). $(C, D)$ Total exploration time (in seconds, sec) of PN1 7 (white bar) and PN24 rats (black bar) for both familiar and moved object at: $(C)$ training and (D) 1 min test. Data are expressed as means \pm SEM ( $n=9-10$ per group). (\#\#\#) $P<0.001$ significance for one sample $t$-tests comparing each group to chance performance (50\%).

To determine whether the nOL memory is rapidly forgotten, different groups of PN17 and PN24 rats were trained and tested $2 \mathrm{~h}$ after training (Fig. 3). We chose this testing time point because Anderson et al. (2004) reported that PN17 rats have no memory for novel object recognition when tested $2 \mathrm{~h}$ after training, in line with the infantile amnesia, whereas at this time point adult rats express significant memory retention.

During nOL training, both PN17 and PN24 rats explored the objects at chance level [one samples $t$-test comparing: PN17 versus chance $(50 \%), t_{(7)}=0.19 P=0.86$ and PN24 versus chance $(50 \%)$, $\left.t_{(8)}=0.71 P=0.50\right]$ (Fig. 3A). At the $2 \mathrm{~h}$ post-training test, PN24 rats had significant memory, as shown by their preferential exploration of the moved object $(65.8 \% \pm 3.8 \%)$ over chance [one samples $t$-test comparing PN24 versus chance $(50 \%), t_{(8)}=4.18 P=$ $0.0031]$. In contrast, the rats trained at PN17 had no memory $(53.2 \% \pm 4.1 \%)$ as revealed by their near chance exploration of both object locations [one samples $t$-test comparing PN17 versus chance (50\%), $t_{(7)}=0.79 P=0.46$ ] (Fig. 3B). Statistical analyses revealed that there was a significant difference in the $2 \mathrm{~h}$ retention test of PN17- and PN24-trained rats [unpaired two-tailed Student's $t$-test comparing \% preference of PN17 versus PN24 at $2 \mathrm{~h}$ Test, $P=0.039$ ] (Fig. 3B). This difference was not due to change in total exploration time because the total time spent exploring the objects did not differ across the two age groups during either training (Fig. 3C) or testing (Fig. 3D) [unpaired two-tailed Student's $t$-test comparing PN17 versus PN24 at: training $(P=0.64)$ and at 2 $\mathrm{h}$ test $(P=0.49)]$.

Thus, these results extend the rapid forgetting of infantile learning to nOL, a nonaversive, hippocampal-dependent task, in rats. 

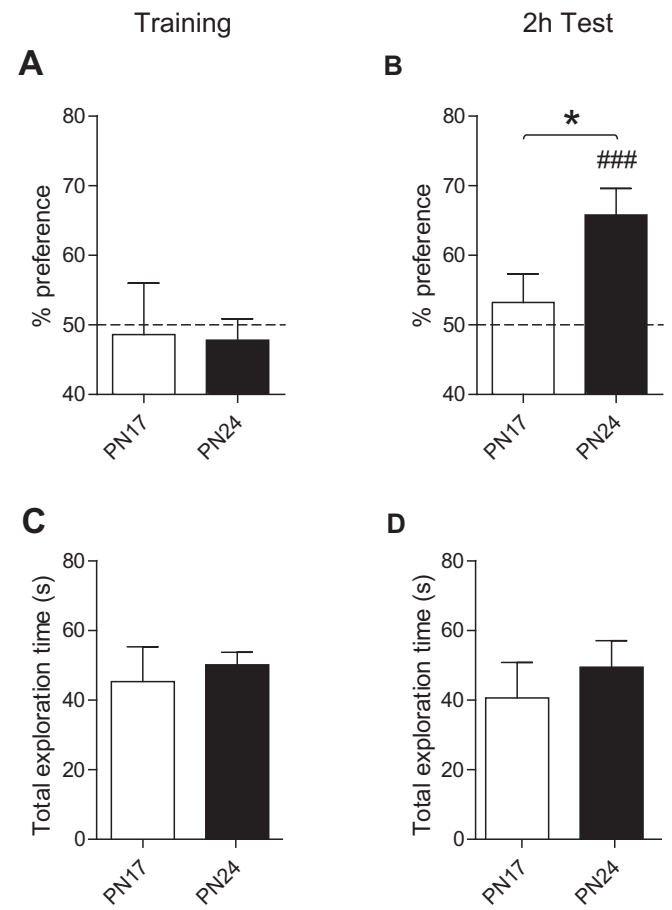

Figure 3. Rats trained at $\mathrm{PN17}$, but not at $\mathrm{PN24}$, show rapid decay in nOL memory retention. $(A, B)$ Percent exploration time of the moved object for PN17 (white bar) and PN24 (black bar) rats at: $(A)$ training and $(B) 2 \mathrm{~h}$ after training (2 h Test). (C,D) Total exploration time (in seconds, sec) of PN1 7 (white bar) and PN24 (black bar) rats for both familiar and moved object at: $(C)$ training and $(D) 2 \mathrm{~h}$ test. Data are expressed as means $\pm \operatorname{SEM}\left(n=8-9\right.$ per group). $\left(^{*}\right) P<0.05$ significance for unpaired two-tailed Student's $t$-test comparing PN17 versus PN24. (\#\#\#) $P<0.001$ significance for one sample $t$-tests comparing each group to chance performance (50\%).

\section{Reminders reinstate the apparently forgotten $\mathrm{nOL}$ memory}

Several studies in human and animal models have shown that reminders of early and apparently forgotten experiences can reinstate the memory (Campbell and Jaynes 1966; Spear and Parsons 1976; Davis and Rovee-Collier 1983; Travaglia et al. 2016a; Guskjolen et al. 2017). Studies done in nonhuman animal models, including ours, have, however, mostly investigated aversive types of learning. Here we tested whether, like for human memories (Davis and Rovee-Collier 1983), nonaversive infantile memories that are apparently forgotten are accessible to reminders and can be reinstated later in life.

One group of rats was trained in nOL at PN17, whereas the other group was used as a control and handled in parallel, but remained in their homecage during the training session. Rats trained at PN17 explored both the objects at chance level [one samples $t$-test comparing: PN17 versus chance $\left.(50 \%), t_{(7)}=0.12 P=0.91\right]$ (Fig. 4A). Three days later, all rats were exposed to a reminder session, which consisted of a $90 \mathrm{sec}$ reexposure, in the training context, to the same objects in the same position that was experienced during training. During this reminder session, both trained and control rats explored both objects at chance level [one samples $t$-test comparing: PN17 trained versus chance $(50 \%), t_{(7)}=0.25 P$ $=0.81$; PN17 control versus chance $(50 \%), t_{(8)}=0.14 P=0.89$ ] (Fig. 4B). However, when the animals were tested 1 min after the reminder, rats trained at PN17 showed significant memory, as in fact they preferentially explored the moved object $(67.0 \% \pm$ $4.8 \%$ ) over chance [one samples $t$-test comparing PN17 trained ver- sus chance (50\%), $t_{(8)}=3.53 P=0.0096$ ] (Fig. 4C). Control rats, as expected, had no memory $(49.4 \% \pm 5.4 \%)$ and in fact explored both the objects at near chance level [one samples $t$-test comparing PN17 control versus chance $(50 \%), t_{(8)}=0.12 P=0.91$ ] (Fig. 4C). Comparing the test performance after the reminder of trained versus control rats revealed a significant difference [unpaired twotailed Student's $t$-test comparing \% preference of trained versus controls at $1 \mathrm{~min}$ Test after reminder $(P=0.028)]$ (Fig. 4C). This difference was not due to change in total exploration time because the total time spent exploring the objects did not differ between the two groups during either the reminder or testing [unpaired twotailed Student's $t$-test comparing PN17 trained versus PN17 control at: reinstatement $(P=0.79)$ and at test $(P=0.56)]$ (Fig. 4D-F). We concluded that the infantile, nonaversive nOL memory, although apparently forgotten, is stored in a latent form, and that reminders can reinstate the memory at later times.

\section{Learning at PN17 is accompanied by a significant switch in hippocampal GluN2B and GluN2A levels}

We have previously found that IA training at PN17 is accompanied by a significant switch in the ratio of the NMDA receptor GluN2B and GluN2A subunit level in the dorsal hippocampus (Travaglia et al. 2016a). A similar switch of the dominant subunit from GluN2B to GluN2A had been described as a mechanism of excitatory synapses maturation occurring in response to experience or stimulation during the developmental critical period of the visual cortex or in young hippocampal slices (Carmignoto and Vicini 1992; Quinlan et al. 1999; Bellone and Nicoll 2007; Matta et al. 2011).

Using Western blot analyses, here we examined whether nOL learning at PN17 changes GluN2B and GluN2A expression levels in the dorsal hippocampus. Rats were euthanized $24 \mathrm{~h}$ after training, a time point when the IA training-induced NMDA receptor subunits switch peaks (Travaglia et al. 2016a). Controls consisted of rats exposed to the arena without objects and euthanized at matched time point. Compared to controls, rats trained at PN17 had a significant increase in both GluN2A (Control $100.0 \pm$ 10.21, Trained $=161.7 \pm 14.95 ; t=3.4 \mathrm{df}=16 ; P=0.0036)$ and GluN2B (Control 100.0 \pm 8.06 , Trained 123.3 $\pm 4.65 ; t=2.34 \mathrm{df}=$ $16 ; P=0.033$ ) (Fig. 5). However, the increase in GluN2A was larger, hence leading to a significant rise in the GluN2A/2B ratio (Control $100.0 \pm 7.3$, Trained 132.4 $\pm 11.73 ; t=2.34 \mathrm{df}=16 ; P=0.032$ ) (Fig. $5)$. These data indicated that, as with IA memory, nOL memory formation at PN17 is accompanied by a significant switch in the ratio of GluN2A/Glu2B expression in the hippocampus.

\section{Injection of BDNF in the dorsal hippocampus immediately after training at PN17 leads to lasting nOL memory}

In the visual cortex BDNF overexpression is sufficient to close the critical period by promoting structural maturation of cortical circuitry and precocious increase of visual acuity (Hanover et al. 1999; Huang et al. 1999). Similar, recombinant BDNF injected bilaterally into the dorsal hippocampus of PN17 rats at the time of IA training is sufficient to promote functional competence and closure of the critical period of rapid forgetting; in fact BDNFinjected rats have long-term and persistent memory expression (Travaglia et al. 2016a).

Here we examined whether, like with IA, a bilateral injection of BDNF into the dorsal hippocampus immediately after nOL training at PN17 is sufficient to promote the expression of a persistent memory.

As in our previous experiment (Fig. 3B), at $2 \mathrm{~h}$ after training, vehicle-injected PN17 rats had no significant memory for the moved object $(51.6 \% \pm 3.8 \%)$, and explored both the objects at 


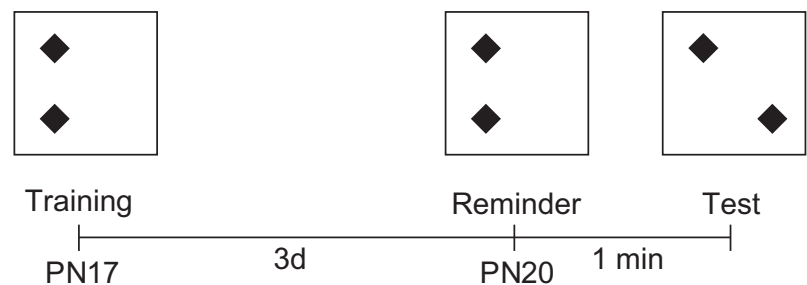

Training

A

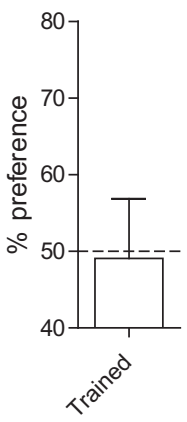

D

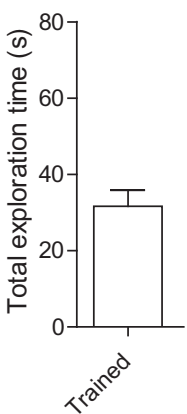

Reminder

B

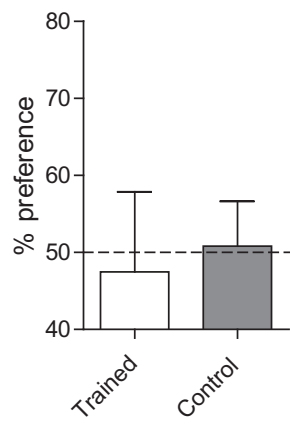

E

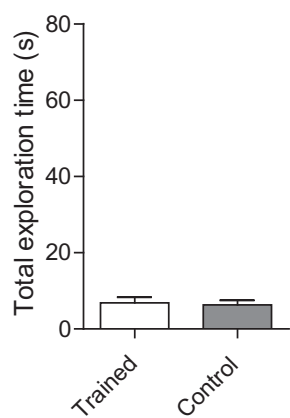

Test

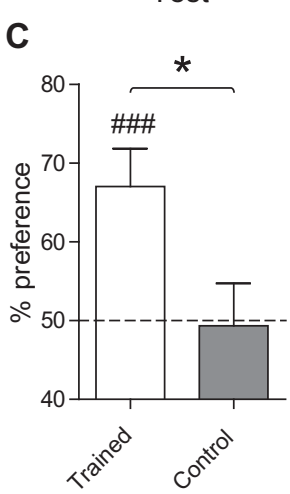

$\mathbf{F}$

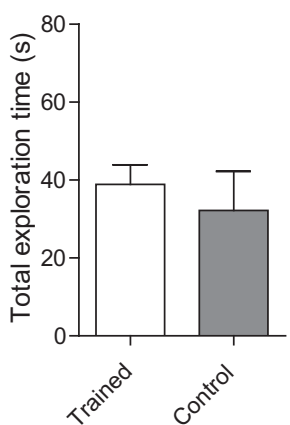

Figure 4. A reminder reinstates the apparently forgotten nOL memory. Experimental schedule is shown above the panels. Rats were handled, habituated to the arena and trained in nOL at PN17 (trained, white bar), or handled, habituated but left in their homecage during the training session (control, gray bar). $(A-C)$ Percent exploration time of the moved object during: $(A)$ training at PN17, $(B)$ reminder at PN20, and $(C)$ test 1 min thereafter. ( $D-F)$ Total exploration time (in seconds, sec) for both familiar and moved object at: $(D)$ training, $(E)$ reminder at PN20, and $(F)$ test 1 min thereafter. Data are expressed as means \pm SEM ( $n=7-8$ per group). $\left(^{*}\right) P<0.05$ significance for unpaired two-tailed Student's $t$-test comparing PN17 trained with PN1 7 control. (\#\#\#) $P<0.001$ significance for one sample $t$-tests comparing each group to chance performance (50\%).

near chance level [one samples $t$-test comparing PN17-veh versus chance $(50 \%), t_{(10)}=0.42 P=0.69$ ] (Fig. 6A,B). In contrast, BDNF-injected rats had significant memory retention, as revealed by the preferential exploration of the moved object $(72.7 \% \pm$ $3.9 \%)$ over chance level [one samples $t$-test comparing PN17BDNF versus chance $\left.(50 \%), t_{(12)}=5.83 P<0.0001\right]$ (Fig. 6B). Hence, BDNF administration produced a long-lasting memory in rats trained at PN17 (unpaired two-tailed Student's $t$-test comparing \% preference of PN17-veh versus PN17-BDNF at $2 \mathrm{~h}$ Test $[P=$ 0.0009]) (Fig. 6B). The increased retention was not due to alteration of total exploration time, since BDNF did not affect the total time spent exploring the objects (unpaired two-tailed Student's $t$-test exploration time of PN17-veh versus PN17-BDNF at $2 \mathrm{~h}$ Test $[P=$ 0.40]) (Fig. 6C,D).

Together, these data led us to conclude that BDNF administration in the dorsal hippocampus of infant rats immediately after training promotes persistent memory retention thus accelerates the closure of the critical period.

\section{Discussion}

Identifying the biological changes occurring in the brain after learning in early childhood is key for understanding how the learning and memory systems build, and is likely to provide fundamental information for understanding the etiology of learning disabilities and developmental psychopathologies.

Here, we showed that, similar to novel object recognition (Anderson et al. 2004; Reger et al. 2009), water maze (Guskjolen et al. 2017), and aversive/ threat-based tasks (Campbell and Spear 1972; Callaghan et al. 2014; Travaglia et al. 2016a), nOL memories in infant rats are very rapidly forgotten. All together, these data indicate that different types of hippocampal-dependent memories occurring in infancy undergo the phenomenon of rapid forgetting associated with infantile amnesia.

Our results extend to nOL the conclusions of other studies on novel object recognition indicating that infant rodents can learn and perform nonaversive incidental learning: Jablonski et al. (2013) and Reger et al. (2009) showed that periweanlings rats are able to recognize a familiar object 15 min and $1 \mathrm{~h}$ after training. Anderson et al. (2004) reported that PN18 rats have memory for the novel object recognition task when tested immediately after training but not $2 \mathrm{~h}$ later, and Krüger et al. (2012) reported that PN16 rats are able to acquire and express memory for a nOL when tested 10 min after training. Our conclusions, however, disagree with those of Westbrook et al. (2014), who indicated that PN17 rats have no memory for nOL when tested 5 min after training, and suggested that PN17 rats are unable to acquire and retain memory for nOL. A possible explanation for the negative findings of Westbrook et al. (2014) may be found in differences of training protocols used, such as handling procedures, and/or the lack of immediate test following training.

As shown by our data, rats trained in nOL at PN17 can express the memory only shortly after training, but then rapidly decrease to chance their retention, suggesting that the expression of the learned information does not persist like it does in adult rats. However, similarly to what we previously found with the IA memory (Travaglia et al. 2016a), here we showed that the apparently forgotten nOL memory is not lost, and, in fact is reinstated by reminders. Hence, like IA, the nOL experience encountered during infancy is stored in a latent form. The expression of memory after the reminder is likely the result of reactivation of the original infantile memory trace, and not of new learning, since no memory was found in controls that were exposed to the reminder but had not undergone previous training. 

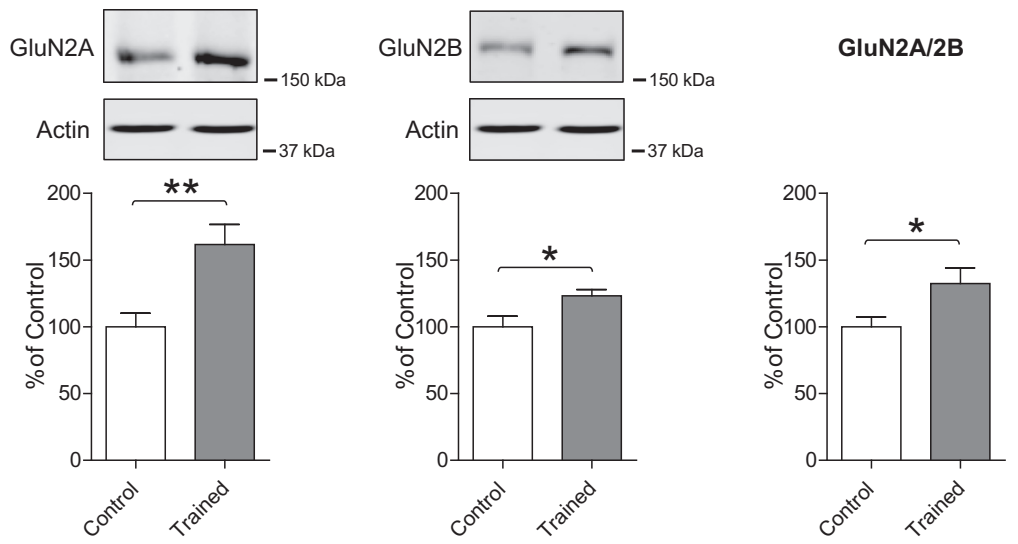

Figure 5. $\mathrm{nOL}$ training at PN17 leads to a significant increase in the GluN2A/GluN2B ratio in the dorsal hippocampus. Examples and densitometric Western blot analyses of dHC total extracts obtained from rats trained in nOL at PN17 (gray bar) and euthanized $24 \mathrm{~h}$ after training. Controls animals were habituated and exposed to the arena, without objects, and euthanized at the matched time point. Data are expressed as mean percentage \pm SEM of control rats. $N=9 /$ group. Unpaired two-tailed Student's $t$-test (*) $P<0.05,\left({ }^{* *}\right) P<0.01$.

Again similarly to IA, nOL training at PN17 induced the regulation of mechanisms that had been previously identified as signature mechanisms of sensory system critical periods and in developing hippocampal slices (Carmignoto and Vicini 1992; Quinlan et al. 1999; Bellone and Nicoll 2007; Matta et al. 2011). First, we found that nOL learning leads to a significant switch in the ratio of GluN2A/GluN2B level in the dorsal hippocampus. Second, we found that the functional maturation of nOL memory expression is promoted by elevating BDNF in the dorsal hippocampus. In fact, in nOL, like in IA (Travaglia et al. 2016a), BDNF bilaterally injected into the $\mathrm{dHC}$ accelerated the closure of the critical period resulting in long-term memory expression. A role of BDNF in accelerating functional maturation and an earlier closure of critical periods was first described in the visual cortex (Hanover et al. 1999; Huang et al. 1999) where BDNF was shown to affect the balance of excitatory and inhibitory neurotransmission (Hanover et al. 1999; Huang et al. 1999). Thus, as during the development of sensory system, BDNF may play a major role in promoting experience-dependent functional maturation of the hippocampal system. In line with this idea, previous data from our laboratory showed that BDNF levels increase over development; in fact, the hippocampus of PN24 and PN80 rats have significantly higher levels of BDNF compared with PN17 rats (Travaglia et al. 2016b). It remains to be determined whether the injection of recombinant BDNF into the hippocampi of PN17 rats brings their BDNF level close to those of PN24 and PN80 rats.

Given that similar mechanisms typically associated with developmental critical periods underlie nonaversive as well as aversive hippocampus-dependent learning and memory during early infancy, we conclude that the hippocampal memory system, like sensory systems, song learning in birds, and language in humans undergoes a developmental critical period (Alberini and Travaglia 2017). We suggest that during this period hippocampal memories are formed, but not expressed long term, because the biological substrates of the hippocampal memory system are still immature. However, as our data on learning-induced switch of levels of NMDAR 2A/2B subunits indicate, the hippocampus matures in response to experience. A critical regulator of this maturation appears to be BDNF, which is sufficient to promote a long-lasting memory when injected immediately after training. Given our data, we suggest that hippocampal functional maturation does not occur by developmental default, but results from experience. In agreement
GluN2A/2B

with this idea, previous data from our laboratory, based on immediate early genes and plasticity markers expression, have shown that the hippocampus at PN17 has significantly higher levels of neuronal activation compared to PN24 and adults (Travaglia et al. 2016b). We speculate that this higher level of hippocampal activation reflects the high level of experience-dependent hippocampal responses in infancy. As we recently discussed (Alberini and Travaglia 2017), we propose that a series of critical periods, subsequently building on each other functional maturation, construct the functional complexity of the hippocampal learning system and the brain. We believe that this complexity in fact emerges sequentially over time through experience, building step-by-step on previously established functional competence.

It is important here to underline that the hippocampus may not be the only region undergoing a critical period maturation in response to hippocampal types of learning, and future studies shall determine whether similar mechanisms of maturation occurs throughout the network of regions functionally connected to the hippocampus.
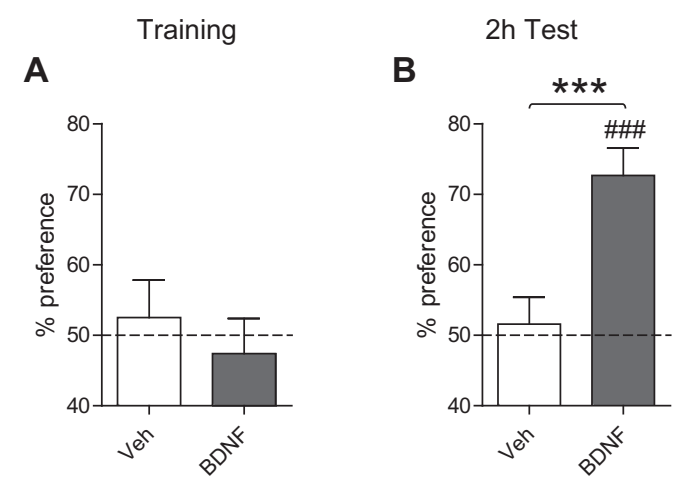

C

D
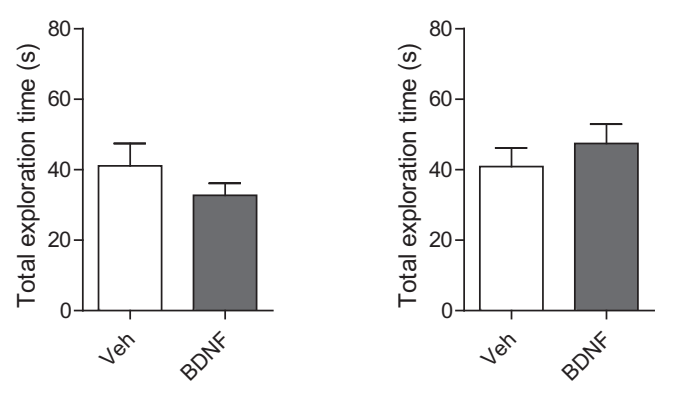

Figure 6. Bilateral injection of BDNF into the dorsal hippocampus immediately after training at PN17 results in persistent nOL memory. $(A, B)$ Percent exploration time of the moved object for PN17 rats injected with vehicle (white) or BDNF (gray bar) at: $(A)$ training and $(B) 2 \mathrm{~h}$ after training (2 h Test). (C,D) Total exploration time (in seconds, sec) of PN17 rats injected with vehicle (white) or BDNF (gray bar) at: (C) training and $(D) 2 \mathrm{~h}$ test. Data are expressed as means \pm SEM $(n=11-13$ per group). $\left.{ }^{* * *}\right) \quad P<0.001$ significance for unpaired two-tailed Student's $t$-test comparing PN17 versus PN24. (\#\#\#) $P<0.001$ significance for one sample $t$-tests comparing each group to chance performance $(50 \%)$. 
In sum, the results of this paper strengthen our conclusion of the existence of a developmental critical period for hippocampusdependent learning and memory. This conclusion has a great deal of important implications in mental health and in diseases. It suggests that, in order to have a balanced and fit development of learning abilities it is critical to be exposed to enriched experiences during infancy and childhood, and that, conversely, impoverishment or deprivation of experience during this early period cause permanent functional limitations.

\section{Materials and Methods}

\section{Animals}

Seventeen- and 24-d-old male and female rats were obtained from pregnant Long Evans female rats (Charles River Laboratories). Rats were housed in $30.80 \mathrm{~cm} \times 40.60 \mathrm{~cm} \times 22.23 \mathrm{~cm}$ plastic cage, containing ALPHA-dri bedding, under a $12 \mathrm{~h}$ light-dark cycle (light on at $07.00 \mathrm{a} . \mathrm{m}$.) with food and water ad libitum. All experiments were carried out during the light cycle. The birth date was considered PNO and the litters were culled to 10-12. Only one male and female per litter was used in any experimental condition. For all the experiments, statistical analyses showed no significant difference when comparing males versus female (unpaired two-tailed Student's $t$-test, $P>0.05)$ Rats were weaned at PN21. All procedures complied with the US National Institute of Health Guide for the Care and Use of Laboratory Animals and were approved by the New York University Animals Care Committees.

\section{Hippocampal cannula implants and injections}

PN15 rat pups were anesthetized with isoflurane mixed with oxygen. Stainless steel cannulas (26-gauge) were implanted bilaterally in the dorsal hippocampus (for PN15, $3.0 \mathrm{~mm}$ anterior, $2.2 \mathrm{~mm}$ lateral and $2.3 \mathrm{~mm}$ ventral from bregma) through holes drilled in the overlying skull. The cannulas were fixed to the skull with dental cement. After recovery from the surgery, pups were returned to the dam and littermates. Hippocampal injections used a 33-gauge needle that extended $1 \mathrm{~mm}$ beyond the tip of the guide cannula and connected via polyethylene tubing to a Hamilton syringe. Injections were delivered at a rate of $0.1 \mu \mathrm{L} \mathrm{min}^{-1}$ using an infusion pump on a total volume of $0.3 \mu \mathrm{L}$ per side over $3 \mathrm{~min}$. The injection needle was left in place for 2 min after the injection to allow complete diffusion of the solution. Recombinant BDNF (PeproTech, cat\# 450-02) was dissolved in $1 \times$ PBS and injected at $0.25 \mu \mathrm{g}$ per side. This dose was shown to rescue the infantile amnesia for IA memory in PN17 rats (Travaglia et al. 2016a).

\section{nOL task}

The nOL task measures the memory of an encountered object's spatial location on the basis of rodents' natural tendency to explore novel stimuli and places (Ennaceur et al. 1997), is nonaversive and dorsal hippocampal-dependent in adult rodents (Mumby et al. 2002; Gaskin et al. 2009; Warburton and Brown 2015). The nOL task was adapted from previously described procedures (Reger et al. 2009; Jablonski et al. 2013), where the object and arena sizes were scaled to the size of the animal within a given age group. Rats were habituated, trained and tested in a square, open field, age-appropriate arena (PN17: $29 \times 29 \times 18 \mathrm{~cm}$; PN24: $44 \times 44 \times 30$ $\mathrm{cm}$ ) with clear Plexiglas walls and floor located in a dim room. Visual cues were provided within the box and on the walls of the room. The walls of the box were covered with white and black paper. One black and one white wall also contained symbols (circle and lines) to create four unique walls. Behavior was recorded with a video camera positioned approximately $1.5 \mathrm{~m}$ above the arena. Rats were first habituated (Hab) to the arena for 5 min each day for 2 consecutive days before training, approximately the same time each day midafternoon (Fig. 1). Twenty-four hours after the last habituation session, each animal was returned to the arena for its training session. Training consisted of exposing the rats to two identical objects constructed from Mega Bloks secured to the floor of the arena. Object sizes were age-appropriate, that is, no taller than twice the size of the rat. Rats were initially placed facing a wall, away from the objects, and were allowed to explore the arena and objects for $5 \mathrm{~min}$. At either $1 \mathrm{~min}$ or $2 \mathrm{~h}$ after training each animal was tested in the arena. During testing, one object remained in the same location as that of training, while the second object was moved to a novel location. Rats were placed in the arena facing the same direction as during training and were allowed to explore for $5 \mathrm{~min}$. Memory reinstatement was carried out by exposing the rats to the training context, with the same objects in the same position, for $90 \mathrm{sec}$. In all experiments, the placement of the novel objects was counterbalanced within each age group and time delay. The arena and objects were cleaned with 70\% ethanol between sessions. Video files were coded and scrambled. An experimenter blind to treatment scored the total time that the rats spent actively exploring each object on each session. For both training and testing phases, exploration was defined as an active event in which the rat was pawing at, sniffing or whisking with its snout directed at the object from a distance of under $\sim 1 \mathrm{~cm}$. Sitting on or next to an object was not counted as active exploration. Memory was measured as the percentage of time spent exploring the object in the novel location compared to the stationary object.

\section{Western blot analysis}

Western blot analysis was carried out as previously reported (Chen et al. 2012). Rats were euthanized, and dorsal hippocampi were rapidly dissected in cold dissection buffer $(2.6 \mathrm{mM} \mathrm{KCl}, 1.23 \mathrm{mM}$ sodium phosphate monobasic, $26 \mathrm{mM}$ sodium bicarbonate, 5 $\mathrm{mM}$ kynurenic acid, $212 \mathrm{mM}$ sucrose, $10 \mathrm{mM}$ dextrose, $0.5 \mathrm{mM}$ $\mathrm{CaCl}_{2}, 1 \mathrm{mM} \mathrm{MgCl}_{2}$ ). Samples were homogenized in ice cold RIPA buffer (50 mM Tris base, $150 \mathrm{mM} \mathrm{NaCl}, 0.1 \%$ SDS, $0.5 \%$ sodium-deoxycholate, $1 \% \mathrm{NP}-40$ ) with protease and phosphatase inhibitors (0.5 mM PMSF, 2 mM DTT, 1 mM EGTA, $2 \mathrm{mM} \mathrm{NaF}, 1$ $\mu \mathrm{M}$ microcystine, $1 \mathrm{mM}$ benzamidine, $1 \mathrm{mM}$ sodium orthovanadate and commercial protease and phosphatase inhibitor cocktails [Sigma-Aldrich]). Protein concentrations were determined using the Bio-Rad protein assay (Bio-Rad Laboratories). Equal amounts of total protein $(20 \mu \mathrm{g}$ per lane) were resolved on denaturing SDS-PAGE gels and transferred to Immobilon-FL transfer membrane (Millipore) by electroblotting. Membranes were dried, reactivated in methanol and washed with water before they were blocked in 5\% (wt) milk and TBS for $1 \mathrm{~h}$ at room temperature $\left(20^{\circ} \mathrm{C}-25^{\circ} \mathrm{C}\right)$. Membranes were then incubated with primary antibody overnight at $4^{\circ} \mathrm{C}$ according to manufacturer's suggestion. Primary antibodies used: GluN2A (1:1,000, Millipore, cat\# 07-632), GluN2B (1:1,000, cat\# 5580, Cell Signaling Technology). The membranes were then washed TBS with $0.2 \%$ Tween 20 (TBST) and incubated with a species-appropriate fluorescently conjugated secondary antibody (goat anti-mouse IRCye 680LT [1:10,000] or goat anti-rabbit IRDye $800 \mathrm{CW}[1: 10,000]$ from LI-COR Bioscience [Lincoln, NE]) for $1 \mathrm{~h}$ at room temperature. Membranes were again washed in TBST and scanned using the Odyssey Infrared Imaging system (LI-COR). Data were quantified using pixel intensities with the Odyssey software according to the protocols of the manufacturer (LI-COR). Actin (1:20,000, cat\# sc-47778, Santa Cruz Biotechnology) was used to costain all membranes and used as loading control for all markers.

\section{Statistical analyses}

Data were analyzed with the Prism 5 (GraphPad Software Inc.). Statistical power calculations have been performed, using the statistical software $G^{*}$ Power. A minimum final group size of about eight rats is required to have a probability of detecting significant effects for behavior experiments. One-sample $t$-tests were used to determine changes in nOL preference compared to chance (50\%). Object location data (percent preference and total times spent exploring each object location) were analyzed with paired Student's t-tests (two-tailed). The significance of the results was accepted at $P<0.05$. 


\section{Acknowledgments}

This work was supported by MH065635 and MH074736 grants to C.M.A. We thank the OVR staff at New York University for technical support.

\section{References}

Akers KG, Martinez-Canabal A, Restivo L, Yiu AP, De Cristofaro A, Hsiang HL, Wheeler AL, Guskjolen A, Niibori Y, Shoji H. 2014. Hippocampal neurogenesis regulates forgetting during adulthood and infancy. Science 344: 598-602.

Alberini CM, Travaglia A. 2017. Infantile amnesia: a critical period of learning to learn and remember. J Neurosci 37: 5783-5795.

Anderson MJ, Barnes GW, Briggs JF, Ashton KM, Moody EW, Joynes RL, Riccio DC. 2004. Effects of ontogeny on performance of rats in a novel object-recognition task. Psychol Rep 94: 437-443.

Bellone C, Nicoll RA. 2007. Rapid bidirectional switching of synaptic NMDA receptors. Neuron 55: 779-785.

Callaghan BL, Li S, Richardson R. 2014. The elusive engram: what can infantile amnesia tell us about memory? Trends Neurosci 37: 47-53.

Campbell BA, Campbell EH. 1962. Retention and extinction of learned fear in infant and adult rats. J Comp Physiol Psychol 55: 1-8.

Campbell BA, Jaynes J. 1966. Reinstatement. Psychol Rev 73: 478-480.

Campbell BA, Spear NE. 1972. Ontogeny of memory. Psychol Rev 79: $215-236$

Carmignoto G, Vicini S. 1992. Activity-dependent decrease in NMDA receptor responses during development of the visual cortex. Science 258: 1007-1011.

Chen DY, Bambah-Mukku D, Pollonini G, Alberini CM. 2012. Glucocorticoid receptors recruit the CaMKII $\alpha$-BDNF-CREB pathways to mediate memory consolidation. Nat Neurosci 15: 1707-1714.

Davis JM, Rovee-Collier CK. 1983. Alleviated forgetting of a learned contingency in 8-week-old infants. Dev Psychol 19: 353-365.

Dumas T, Rudy J. 2010. In Oxford Handbook of Developmental Behavioral Neuroscience. Oxford University Press, New York.

Ennaceur A, Neave N, Aggleton JP. 1997. Spontaneous object recognition and object location memory in rats: the effects of lesions in the cingulate cortices, the medial prefrontal cortex, the cingulum bundle and the fornix. Exp Brain Res 113: 509-519.

Gaskin S, Gamliel A, Tardif M, Cole E, Mumby DG. 2009. Incidental (unreinforced) and reinforced spatial learning in rats with ventral and dorsal lesions of the hippocampus. Behav Brain Res 202: 64-70.

Gerstein H, Hullinger R, Lindstrom MJ, Burger C. 2013. A behavioral paradigm to evaluate hippocampal performance in aged rodents for pharmacological and genetic target validation. PLoS One 8: e62360.

Guskjolen A, Josselyn SA, Frankland PW. 2017. Age-dependent changes in spatial memory retention and flexibility in mice. Neurobiol Learn Mem 143: 59-66.

Hanover JL, Huang ZJ, Tonegawa S, Stryker MP. 1999. Brain-derived neurotrophic factor overexpression induces precocious critical period in mouse visual cortex. J Neurosci 19: Rc40.

Hayne H. 2004. Infant memory development: Implications for childhood amnesia. Dev Rev 24: 33-73.

Heim C, Nemeroff CB. 2001. The role of childhood trauma in the neurobiology of mood and anxiety disorders: preclinical and clinical studies. Biol Psychiatry 49: 1023-1039.
Hensch TK. 2005. Critical period plasticity in local cortical circuits. Nat Rev Neurosci 6: 877-888.

Huang ZJ, Kirkwood A, Pizzorusso T, Porciatti V, Morales B, Bear MF, Maffei L, Tonegawa S. 1999. BDNF regulates the maturation of inhibition and the critical period of plasticity in mouse visual cortex. Cell 98: 739-755.

Jablonski SA, Schreiber WB, Westbrook SR, Brennan LE, Stanton ME. 2013. Determinants of novel object and location recognition during development. Behav Brain Res 256: 140-150.

Kim JH, McNally G, Richardson R. 2006. Recovery of fear memories in rats: role of gamma-amino butyric acid (GABA) in infantile amnesia. Behav Neurosci 120: $40-48$.

Krüger HS, Brockmann MD, Salamon J, Ittrich H, Hanganu-Opatz IL. 2012. Neonatal hippocampal lesion alters the functional maturation of the prefrontal cortex and the early cognitive development in pre-juvenile rats. Neurobiol Learn Mem 97: 470-481.

Li S, Callaghan BL, Richardson R. 2014. Infantile amnesia: forgotten but not gone. Learn Mem 21: 135-139.

Matta JA, Ashby MC, Sanz-Clemente A, Roche KW, Isaac JT. 2011. mGluR5 and NMDA receptors drive the experience- and activity-dependent NMDA receptor NR2B to NR2A subunit switch. Neuron 70: 339-351.

Mumby DG, Gaskin S, Glenn MJ, Schramek TE, Lehmann H. 2002. Hippocampal damage and exploratory preferences in rats: memory for objects, places, and contexts. Learn Mem 9: 49-57.

Nelson C. 2000. Neural plasticity and human development: the role of early experience in sculpting memory systems. Dev Sci 3: 115-136.

Quinlan EM, Philpot BD, Huganir RL, Bear MF. 1999. Rapid, experiencedependent expression of synaptic NMDA receptors in visual cortex in vivo. Nat Neurosci 2: 352-357.

Reger ML, Hovda DA, Giza CC. 2009. Ontogeny of rat recognition memory measured by the novel object recognition task. Dev Psychobiol 51: 672-678.

Rovee-Collier C. 1999. The development of infant memory. Curr Dir Psychol Sci 8: 80-85.

Rudy JW, Morledge P. 1994. Ontogeny of contextual fear conditioning in rats: implications for consolidation, infantile amnesia, and hippocampal system function. Behav Neurosci 108: 227-234.

Schiffino FL, Murawski NJ, Rosen JB, Stanton ME. 2011. Ontogeny and neural substrates of the context preexposure facilitation effect. Neurobiol Learn Mem 95: 190-198.

Spear N, Parsons P. 1976. Analysis of a reactivation treatment: Ontogenetic determinants of alleviated forgetting. Lawrence Erlbaum Associates Hillsdale, New Jersey.

Travaglia A, Bisaz R, Sweet ES, Blitzer RD, Alberini CM. 2016a. Infantile amnesia reflects a developmental critical period for hippocampal learning. Nat Neurosci 19: 1225-1233.

Travaglia A, Bisaz R, Cruz E, Alberini CM. 2016b. Developmental changes in plasticity, synaptic, glia and connectivity protein levels in rat dorsal hippocampus. Neurobiol Learn Mem 135: 125-138.

Warburton EC, Brown MW. 2015. Neural circuitry for rat recognition memory. Behav Brain Res 285: 131-139.

Westbrook SR, Brennan LE, Stanton ME. 2014. Ontogeny of object versus location recognition in the rat: acquisition and retention effects. Dev Psychobiol 56: 1492-1506.

Received October 29, 2017; accepted in revised form January 12, 2018. 


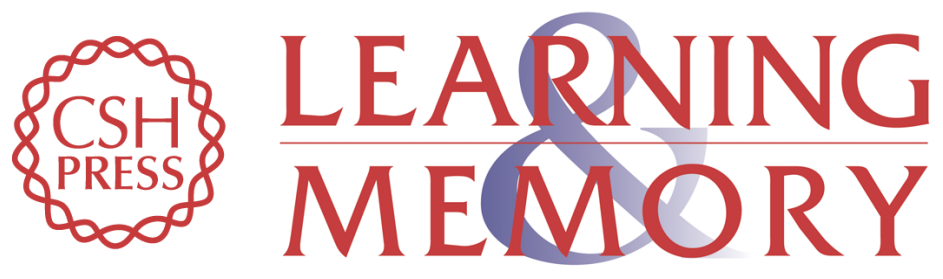

\section{Mechanisms of critical period in the hippocampus underlie object location learning and memory in infant rats}

Alessio Travaglia, Adam B. Steinmetz, Janelle M. Miranda, et al.

Learn. Mem. 2018, 25:

Access the most recent version at doi:10.1101/Im.046946.117

References This article cites 36 articles, 5 of which can be accessed free at:

http://learnmem.cshlp.org/content/25/4/176. full.html\#ref-list-1

Creative This article is distributed exclusively by Cold Spring Harbor Laboratory Press for the

Commons

first 12 months after the full-issue publication date (see

License http://learnmem.cshlp.org/site/misc/terms.xhtml). After 12 months, it is available under a Creative Commons License (Attribution-NonCommercial 4.0 International), as described at http://creativecommons.org/licenses/by-nc/4.0/.

Email Alerting Receive free email alerts when new articles cite this article - sign up in the box at the Service top right corner of the article or click here. 